CASE REPORT

$02 / 04 / 2020$

$1(1): 1-10$

DOI: https://doi.org/10.46295/1:1.LOPHO

\title{
Lophomoniasis associates with chronic immune compromise and respiratory coinfections: a case report.
}

Erick E. Escalante-Díaz1,2, Ariadna Areli Martínez-Salinas', Mario F. Gómez Núñez ${ }^{1,3}$, Rozana Reyes-Gámez, Lucero A. Torres-Alarcón ${ }^{1,3}$ \& Alberto N. Peón 1,4,*

${ }^{1}$ Sociedad Española de Beneficencia, Pachuca, Hidalgo.

2 Benemérita Universidad Autónoma de Puebla (BUAP), Facultad de Medicina.

${ }_{3}^{3}$ Universidad Autónoma del Estado de Hidalgo (UAEH), Área Académica de Medicina.

4 Escuela Superior de Apan (ESAp), UAEH.

*Corresponding author.

Abstract

Lophomoniasis is a respiratory tract infection produced by a protozoon that is associated with the gastrointestinal tract of cockroaches and thermites. Few cases have been reported in the world, in such a way that we lack much knowledge about the disease. Hereby we present a case of a chronic Lophomonas spp infection that associates with a strong respiratory, renal and hepatic dysfunction that are manifested by multiple alterations in data from hematic biometrics. Importantly, the infection induces a strong and chronic immune hypo-responsiveness that persists after parasite's clearance and associates with the acquisition of respiratory nosocomial infections.

Highlights:

1) Lophomoniasis produces dysfunctions in gas interchange that relates with lung infiltration.

2) Lophomoniasis relates with the elevation of renal and hepatic failure markers.

3) Lophomoniasis associates with longlasting immune hyporesponsiveness that may facilitate further respiratory infections.
Keywords:

Lophomoniasis, Lophomonas spp, immune regulation, respiratory dysfunction, nosocomial infections.

Correspondence should be addressed to: investigacion@benepachuca.com 
SÖCIÉDÁAD

ESPAÑOLA DEBNEFICENCIA AC

P A C H U C A
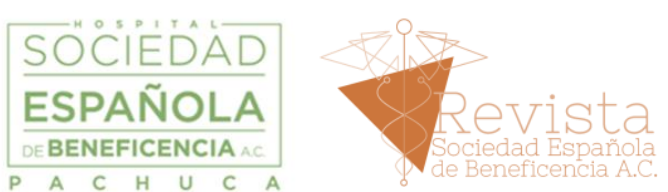

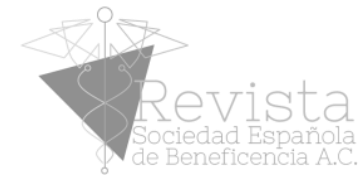

\section{Introduction}

Lophomonas spp is a pluriflagellated protozoon that has been described as a commensal of the gastrointestinal tract (GI) of thermites and cockroaches, where it aids its hosts to digest lignocellulosic materials (1). It is known that this protozoon is able to encyst itself in order to withstand the atmospheric conditions when it leaves the host's GI, but it is only speculated that such cysts may be able to infect the human respiratory tract (RT) upon direct inhalation (2), or even by the ingestion of foods that have been contaminated with thermite or cockroach feces. It is hypothesized that the temperature and humidity of the RT provides adequate conditions for trophozoite excystation (3), which is thought to be the beginning of a respiratory pathology known as lophomoniasis.

Trophozoite reproduction occurs mainly by binary fission within the host's RT, and upon excystation the preferred site for the infection is the respiratory tract, but paranasal sinus (4) and urinary tract infections (5) have also been reported.

The signs and symptoms of the disease are strongly unspecific (including cough, sputum, dyspnea, fever and chest pain) (6), so that lophomoniasis is sometimes confused with virus or bacteria-induced pneumonias, bronchial asthma, bronchiectasis or pulmonary abscesses (2). Its correct diagnosis depends upon the microscopic observation of samples of sputum, bronchial brushings or secretions, tracheal aspirates, or bronchoalveolar lavages. In such tests the protozoon appears as unipolarlly pluriflagellated round, ovoid or piriform cells of 20-60 $\mu \mathrm{m}$ in length and $12-20 \mu \mathrm{m}$ in width, that have a highly granular cytoplasm and an inconspicuous nucleus (2, 7). In order to avoid the aforementioned diagnostic problems, Fakhar, M et al (2019) proposed a polymerase chain reaction (PCR)-based diagnostic technique (8) that is yet to be widely tested. On the other hand, Lophomonas' cysts are spherical or oblong and surrounded by a homogeneous membrane, and may have a torpid metabolism to withstand the lack of nutriments in the environment. As its life cycle has never been directly observed and documented it is not known if lophomoniasis is communicable among people, or if it is exclusively transmitted by zoonosis.

In this way, it is thought that China has the largest number of reported cases because of their traditional consumption of fried cockroaches, which may facilitate the ingestion of the protozoon's cysts by human patients. However, there are no reports that give information about this hypothesis, and about the possibility for the cysts to withstand the high temperatures associated with the frying process.

Lophomoniasis is considered as an emerging disease, as only 61 cases have been confirmed around the world, and the majority of such reports are $\approx 10$ years old (3). Studies about the incidence and prevalence of the disease have never been done in human patients, but Martínez-Girón, R. et al (2017) reported that the 
SOCIÉEAAD

ESPAÑOLA DEBNEFICENCIA AC

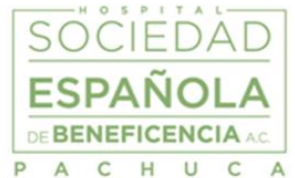

prevalence of L. blattarum in the hindgut of the cockroach Blattella germanica was 13.6\%, at least in one location in Spain (9). Apparently, the infection occurs mainly in men (70.9 \% of the cases), with China being the most affected country, followed by Spain and Peru (2). Nonetheless, in the last 5 years an increasing number of cases have been reported throughout the world, to include India (10, 11) and Mexico (12).

Lophomoniasis' pathogenic mechanisms have not been studied, but other lunginfecting protozoon parasites have been shown to possess varied damageenhancing mechanisms, such as cytoadherence, breakdown of epithelial barriers and apoptosis induction (3). Lophomoniasis usually occurs in patients that have a concomitant RT-disease (10, 13-15), are immunocompromised because of an underlying disease (16) or receive immunosuppressant drugs as a tolerogenic therapy to organ transplantation $(6,17)$. Nonetheless, although sparse, there are also reports of otherwise healthy individuals with such disease (7). In this way, some authors think that Lophomonas spp may have an opportunistic behavior, rather than a full parasitic ability (6), or even that the protozoon is in fact a commensal that can become pathogenic under some unrelated pathological circum-stances (3).

In the present article, we report a case of lophomoniasis, studying the impact that the disease had on respiratory, renal, hepatic and immunologic functions, with aims to provide a deeper understanding on such an emerging disease.

\section{Materials and methods}

2.1 Pulmonary infiltration measurement. Anteroposterior radiographies were taken daily to monitor the growth of lung-infiltrations, and the extent of such infiltrates was analyzed through the use of the free-hand draw tool of AxioVision SE64 software by two different people for each plaque. Measurements were expressed as square pixels.

2.2 Data analysis and statistical analysis. Data from hematic biometry, gasometry and $\mathrm{x}$-rays were plotted and analyzed with GraphPad Prism 5 Software in all cases. The extent of the lung infiltration was presented as mean \pm standard error mean. The significance of the differences between the experimental groups was measured using a two-tailed Student's t-test, considering statistical significance in $\mathrm{P}$ values $<0.05$.

\section{Case presentation}

A 45 years old male patient presented to the hospital after 9 days of fever, cephalea, dyspnea, irritability, thoracic pain, shivers, odynophagia, myalgia, arthralgia, polypnea, purulent respiratory secretions and attack to his general state, reporting the start of a persistent and painful cough with sputum one month before the appearance of the aforementioned signs and symptoms. His 
SÖC̊IÉÁÁ ESPAÑOLA DEBENEFICENCIAAC
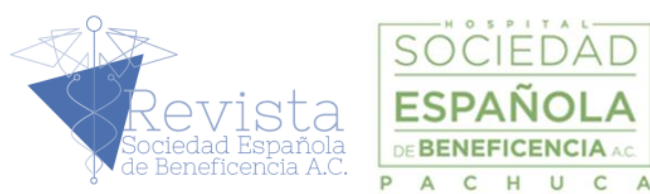

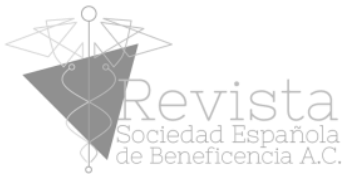

a)

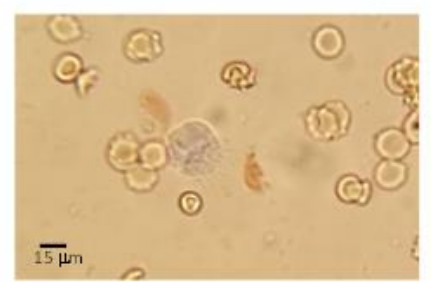

b)

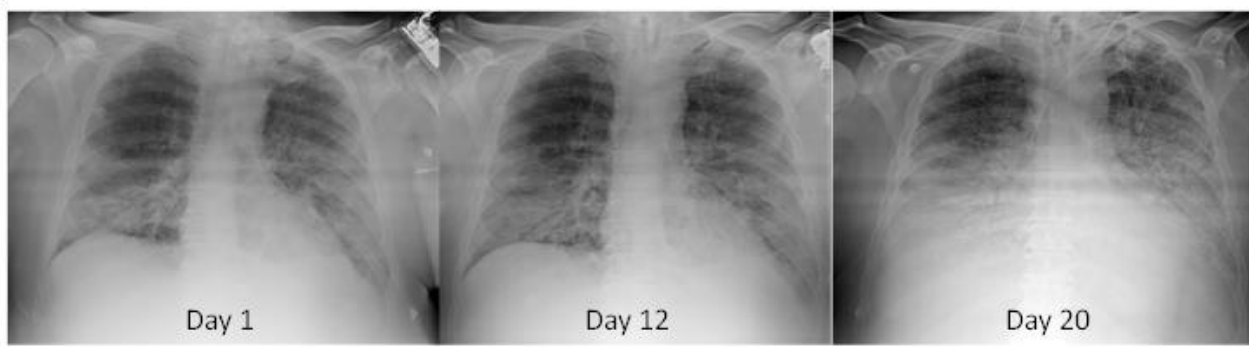

Figure 1. Pulmonary infection with Lophomonas spp. Lophomonas spp. trophozoites were observed in bronchial exudates stained with Nielsen stain (400X magnification) (a), while linear and nodular infiltrates were observed in both lungs along the whole period of the infection (12

days) with the protozoon, and even to day 20 after hospital admission (b). The size of the infiltrates was digitally measured at the days mentioned (c). Infiltrates were measured twice by different people. The statistical significance between groups was determined by two-tailed Student's t-test. * $\mathrm{P}<0.05$.

a)

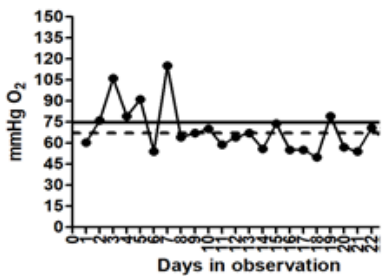

d)

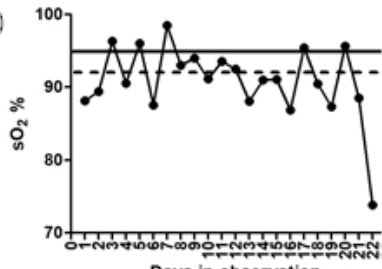

b)

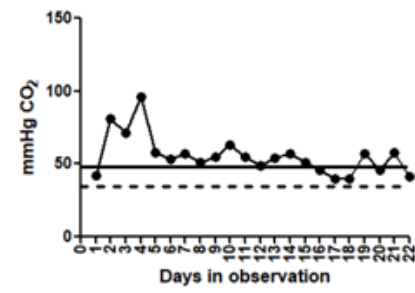

e)

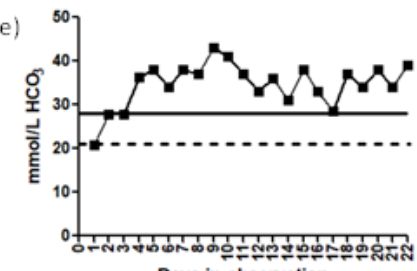

c)

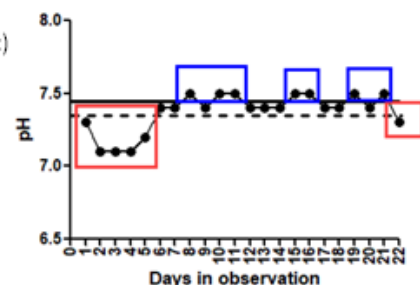

Days in observation Days in observation

Figure 2. Lophomoniasis induces a compromised respiratory function. Oxygen pressure (a) and saturation (b) were measured along with CO2 pressure (c) and serum bicarbonate to determine the extent of respiratory function loss (d), showing alternated episodes of respiratory acidosis (in red squares) with metabolic alkalosis (in blue squares) (e). Solid lines mark the highest healthy level for each parameter, while the dotted lines mark the lowest.

comorbidities included non-controlled type 2 diabetes, morbid obesity, and tobacco consumption. He never received anti-influenza vaccination. As such he was admitted to the hospital in barbituric-induced comma (induced in other health care center) and under treatment with oseltamivir for type-A influenza suspicion. However, an immunochromatographic assay revealed that he was free of such infection. On the other hand, the microscopic examination of a 
SÖC̊IÉDÁAD ESPAÑOLA DEBENEFICENCIAAC

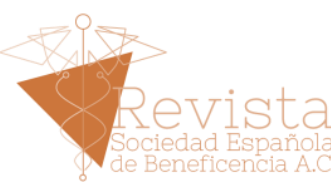

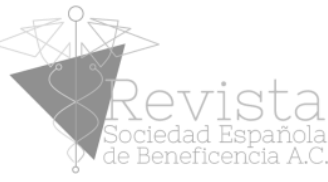

a)

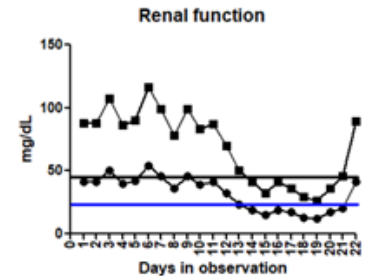

d)

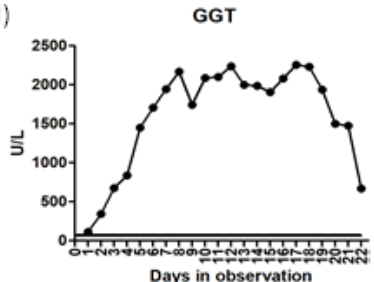

ALP

b)

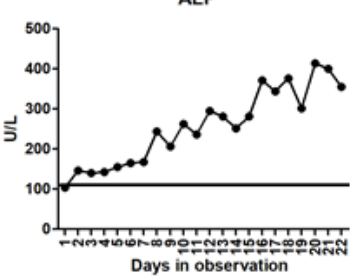

e)

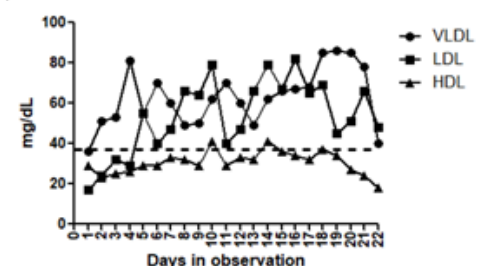

LDH

c)
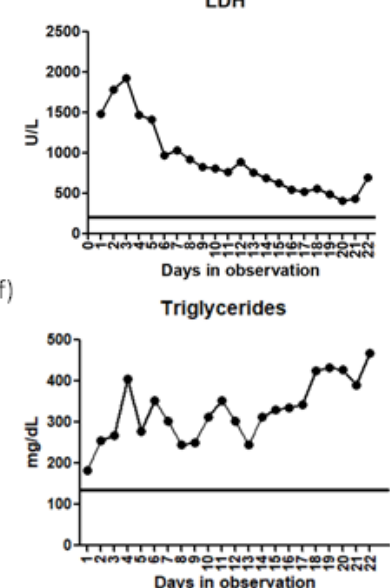

Figure 3. Hepatic and renal dysfunction. Both blood urea nitrogen (BUN) and serum urea (SU) high levels are associated with a decreased renal function (a), while alkaline phosphatase (ALP) (b), lactate dehydrogenase (LDH) (c), and gamma-glutamic transpeptidase (GGT) (d) were used as

markers for hepatic function. On the other hand very low-density lipoproteins (VLDL), lowdensity lipoproteins (LDL), high-density lipoproteins (HDL) (e) and triglycerides (f) were used to asses cardiac risk. Solid lines mark the highest healthy level for each parameter, while the dotted lines mark the lowest. Solid black and solid blue lines mark SU and BUN highest healthy levels, respectively.

a)

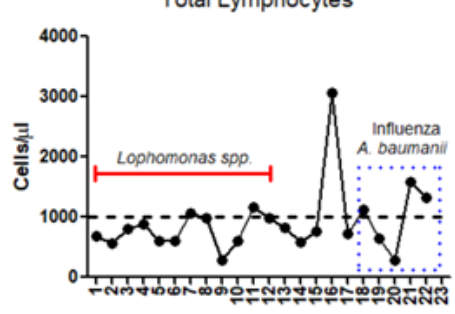

c)

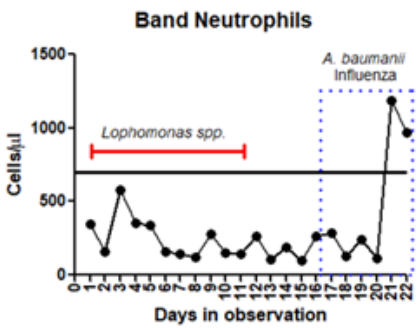

b)

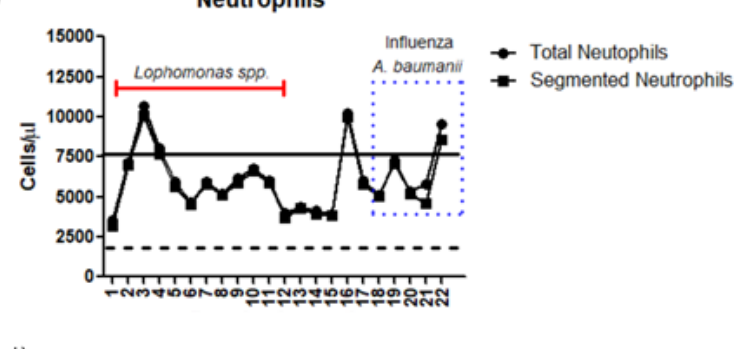

d)

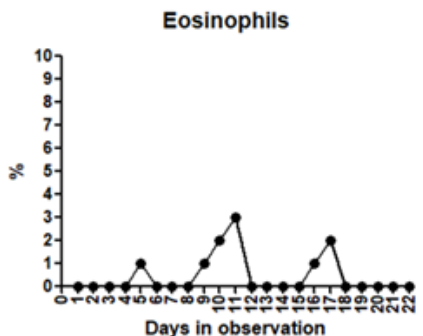

Figure 4. Lophomoniasis associates with chronic immune hypo-responsiveness. Total lymphocytes (a), as well as segmented (b) and band (c) neutrophils were quantified as markers of immune responsiveness. Solid lines mark the highest healthy level for each parameter, while the dotted lines mark the lowest.

bronchial exudate revealed the presence of abundant Lophomonas spp. trophozoites (Fig. 1a), which was confirmed by the observation of linear and nodular infiltrations in $\mathrm{x}$-rays of both lungs (Fig. 1b). 500mg of metronidazole was administered thrice daily until no more trophozoites could be detected in such exudates (12 days later), as this medication had been described as useful in a number of case reports 
SÖCIÉDÁAD

ESPAÑOLA DEBNEFICENCIA AC

P A C H U C A
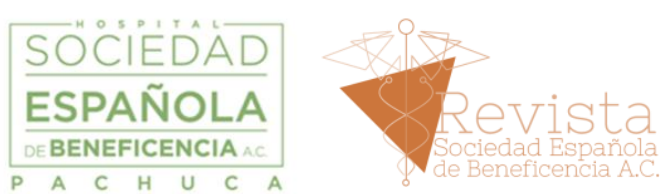
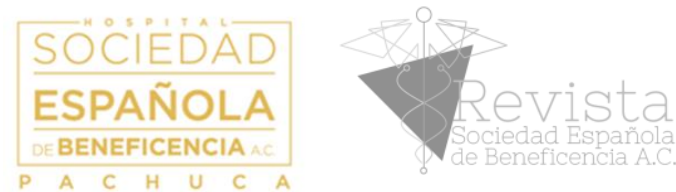

constant gasometry tests, in order to avoid further complications. As for diagnosis, the PCR test developed by Fakhar et al (8) seems to be a good option, but many laboratories may not find suitable to have in stock a test for a rare disease. As a consequence, the diagnosis should be based on the microscopically observation of the protozoon and supported by both radiographies showing nodular and linear infiltrations and a diagnosis for a non-bacterial, nor viral pneumonia.

On the other hand, eukaryotic parasites have been described $(18,19)$ to produce a profound effect on their host's immune response that is mainly oriented to avoid their detection or to deter the effector mechanisms that would otherwise cause their elimination. In this way, the debate about this protozoon being a parasite or an opportunistic would be resolved if we got to know more about its ability to modulate its host's immunity.

In this line of thought it is important to mention that the only trait that has been reported as a common signature in this pathology is a moderate rise in circulating eosinophils, but this trait has only been reported to occur in 35\% of the infected patients (2). We think that a more profound analysis of the hematic biometry parameters may be able to give important data about the immune profile that is elicited by such infection. An example of this is that we did not detect a significant and stable rise in eosinophils in this case.

Moreover, as parasite infections often skew host's immune responses towards a Th2 phenotype and induce regulatory mechanisms that limit protective Th1/Th17 responses, it is common to find that such parasites have the ability to increase host's susceptibility to bacterial, viral and/or fungal infections (20). We think that this may be in line with the fact that this patient presented two different nosocomially acquired lung infections: one of bacterial and one of viral nature. Both coinfections may have been facilitated through the induction of the chronic lymphocyte and neutrophil hypo-responses that we detected during the period when the patient still had lophomoniasis, and afterwards.

As happens with many emerging diseases, the number of studies about lophomoniasis is not high, so that we lack much understanding about its pathophysiology. Moreover, most of such studies are case reports, where the fluctuation of pathological markers is not described. In this way, we think that a deeper inquiry into each case's characteristics is needed to provide more knowledge about the pathological mechanisms of such an emerging disease.

Nonetheless, considering that lophomoniasis usually occurs in patients with concomitant diseases and/or immunosuppressant conditions and medications, it is hard to establish a cause-consequence relationship using data from case reports. Moreover, as Lophomonas spp infection is rare, and most case reports comprise single cases, most observations cannot be statistically processed. Thus, the parasite must be cultured and an in vivo animal model must be developed in order to provide better knowledge about the 
SOCIÉÉÁAD

ESPAÑOLA DEBENEFICENCIA AC
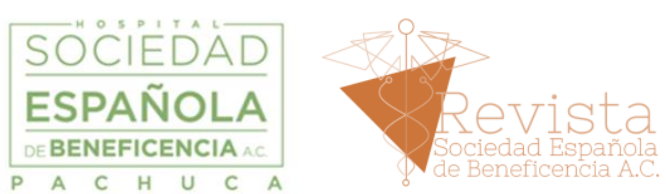

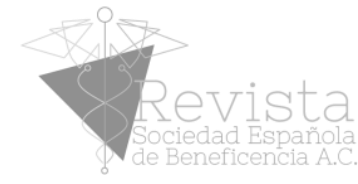

disease's pathophysiological mechanisms.

Taking into account that no epidemiological studies have been done about this disease, and the hypothesis that states that this protozoon may become pathogenic under certain unknown circumstances, it is important not to underestimate the possibility that this disease may have in fact a higher prevalence than what is usually thought. In this line of thought, an epidemiological study may also be needed.

\section{Conclusions}

Lophomonas spp is a protozoon that may infect the human RT trough cyst inhalation, to produce a respiratory disease that is characterized by a defective gas interchange in the lungs, which relates with a high pulmonary infiltration. Although renal and hepatic dysfunctions were also present in this case, no certainty about its causality can be obtained from this single case. Nonetheless, a strong immune compromise was detected in relation with lophomoniasis, manifested by a chronic immune hypo-responsiveness and a higher susceptibility to develop pulmonary coinfections.

6. Conflicts of interest: none of the authors have any conflict of interest to declare.

7. Informed consent: written informed consent was obtained from the patient for publication of this manuscript and any accompanying images.

8. Acknowledgments: the auth-ors wish to thank Sociedad Española de Beneficencia's Hospital for funding this research and its publication, and for the financial aid provided to the students involved in this research. Moreover, this is the first Academic Journal in the areas of Medicine and Biomedicine that has been developed in the State of Hidalgo, being this its first published article. The Journal is entirely sponsored by the aforementioned Institution.

\section{References}

1. Mu XL, Shang Y, Zheng SY, Zhou B, Yu B, Dong XS, et al. [A study on the differential diagnosis of ciliated epithelial cells from Lophomonas blattarum in bronchoalveolar lavage fluid]. Zhonghua jie he he hu xi za zhi = Zhonghua jiehe he huxi zazhi $=$ Chinese journal of tuberculosis and respiratory diseases. 2013;36(9):646-50.

2. Martinez-Giron R, van Woerden HC. Lophomonas blattarum and bronchopulmonary disease. Journal of medical microbiology. 2013;62(Pt 11):1641-8.

3. Martinez-Giron R, van Woerden HC. Bronchopulmonary lophomoniasis: emerging disease or unsubstantiated legend? Parasites \& vectors. 2014;7:284. 


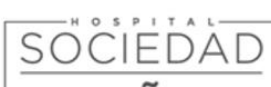

ESPAÑOLA DEBENEFICENCIAAC
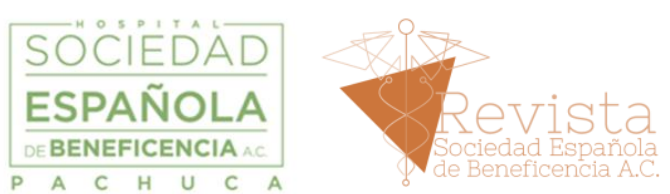

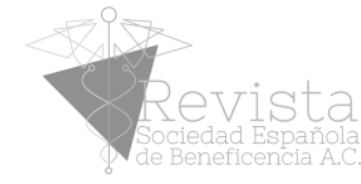

4. Berenji F, Parian M, Fata A, Bakhshaee M, Fattahi F. First Case Report of Sinusitis with Lophomonas blattarum from Iran. Case reports in infectious diseases. 2016;2016:2614187.

5. Xue J, Li YL, Yu XM, Li DK, Liu MF, Qiu JF, et al. Bronchopulmonary infection of Lophomonas blattarum: a case and literature review. The Korean journal of parasitology. 2014;52(5):521-5.

6. Wang Y, Tang Z, Ji S, Zhang Z, Chen J, Cheng Z, et al. Pulmonary Lophomonas blattarum infection in patients with kidney allograft transplantation. Transplant international : official journal of the European Society for Organ Transplantation. 2006;19(12):1006-13.

7. Meng SS, Dai ZF, Wang HC, Li YX, Wei DD, Yang RL, et al. Authenticity of pulmonary Lophomonas blattarum infection: A case report. World journal of clinical cases. 2019;7(1):95-101.

8. Fakhar M, Nakhaei M, Sharifpour A, Kalani H, Banimostafavi ES, Abedi S, et al. First Molecular Diagnosis of Lophomoniasis: the End of a Controversial Story. Acta parasitologica. 2019;64(2):390-3.

9. Martinez-Giron R, Martinez-Torre C, van Woerden HC. The prevalence of protozoa in the gut of German cockroaches (Blattella germanica) with special reference to Lophomonas blattarum. Parasitology research. 2017;116(11):3205-10.

10. Verma S, Verma G, Singh DV, Mokta J, Negi RS, Jhobta A, et al. Dual infection with pulmonary tuberculosis and Lophomonas blattarum in India. The international journal of tuberculosis and lung disease : the official journal of the International Union against Tuberculosis and Lung Disease. 2015;19(3):368-9.

11. Tyagi R, Anand KB, Teple K, Negi RS. Lophomonas blattarum infection in immunocompetent patient. Lung India : official organ of Indian Chest Society. 2016;33(6):667-8.

12. Saldana NG, Mendoza FJO, Larrauri FR, Trujillo DMG, Montoya EV, De La Garza EA, et al. Bronchopulmonary infection by Lophomonas blattarum in a pediatric patient after hematopoietic progenitor cell transplantation: first report in Mexico. Journal of thoracic disease. 2017;9(10):E899-E902.

13. Zerpa R, Ore E, Patino L, Espinoza YA. [Lophomonas sp. in respiratory tract secretions in hospitalized children with severe lung disease]. Revista peruana de medicina experimental y salud publica. 2010;27(4):575-7.

14. Zeng H, Kong X, Chen X, Luo H, Chen P, Chen Y. Lophomonas Blattarum infection presented as acute exacerbation of chronic obstructive pulmonary disease. Journal of thoracic disease. 2014;6(6):E73-6.

15. Thakur C, Verma S, Negi RS, Kumar V, Gupta S, Sharma V. Lophomonas blattarum co-infection in a patient with multidrug-resistant tuberculosis. The international journal of tuberculosis and lung disease : the official journal of the International Union against Tuberculosis and Lung Disease. 2017;21(10):1185-7. 
SÖCIËB́ÁAD

ESPAÑOLA DEBENEFICENCIAAC

16. Vidal C, Barthel E, Rodriguez MLA. [Lung infection by lophomonas spp. in a female patient with acute myeloid leukemia]. Revista peruana de medicina experimental y salud publica. 2018;35(3):527-30.

17. He Q, Chen X, Lin B, Qu L, Wu J, Chen J. Late onset pulmonary Lophomonas blattarum infection in renal transplantation: a report of two cases. Internal medicine. 2011;50(9):1039-43.

18. Terrazas CA, Terrazas LI, Gomez-Garcia L. Modulation of dendritic cell responses by parasites: a common strategy to survive. Journal of biomedicine \& biotechnology. 2010;2010:357106.

19. Peon AN, Ledesma-Soto Y, Terrazas Li. Regulation of immunity by Taeniids: lessons from animal models and in vitro studies. Parasite immunology. 2016;38(3):124-35.

20. Salgame P, Yap GS, Gause WC. Effect of helminth-induced immunity on infections with microbial pathogens. Nature immunology. 2013;14(11):1118-26. 